\title{
Análisis del efecto del cambio en el uso del suelo sobre el escurrimiento en la cuenca alta del río Sauce Chico, Argentina
}

\section{Use changes effect analysis on runoff in the upper basin of the Sauce Chico River, Argentina}

\author{
Lucía De Antueno; Fernanda J. Gaspari y Adriana Guzmán Guaraca² \\ ${ }^{1}$ Curso de Manejo de Cuencas Hidrográficas -Centro de Estudios Integrales de la \\ Dinámica Exógena (CEIDE). Facultad de Ciencias Agrarias y Forestales. Universidad \\ Nacional de La Plata. \\ ${ }^{2}$ Becaria Doctoral CICPBA. \\ Dirección: Diagonal 113 № 469. La Plata, CP 1900, Buenos Aires. Argentina. \\ E-mail: cuencas@agro.unlp.edu.ar
}

De Antueno, L., Gaspari, F. J. \& Guzmán Guaraca, A. (2020). Análisis del efecto del cambio en el uso del suelo sobre el escurrimiento en la cuenca alta del río Sauce Chico, Argentina. Revista Estudios Ambientales, 8(1), 20-42. 


\section{RESUMEN}

Los cambios de cobertura y uso del suelo son reconocidos como una de las principales influencias en la dinámica hídrica superficial. La modelización hidrológica permite comparar los escenarios pluviales y de cambio de uso del suelo en cuencas hidrográficas. El objetivo propuesto fue analizar el efecto de la cobertura y uso del suelo sobre la generación de escorrentía en la cuenca alta de río Sauce Chico (CARSCH), Argentina. Se desarrolló una metodología geoespacial de análisis de la situación morfométrica como herramienta de base para la modelización hidrológica, que se realizó en HEC-HMS 4.2aplicando el modelo Número de curva (NC)para un evento pluvial extremo y otro modal con fines de comparación de la respuesta de la cuenca. La zonificación de la cobertura vegetal se definió a través de un geoprocesamiento digital, para cuatro escenarios de uso de suelo, considerándolos como representativos de una tendencia de cambio espacio temporal. Al analizar los resultados se determinó que el mayor volumen escurrido y caudales máximos en tormentas extremas se generan en áreas serranas, mientras que ante eventos modales los máximos se registran en zonas de lomadas. Además, los resultados revelan que el cambio en el uso del suelo tiene una influencia más significativa en el comportamiento de los caudales ante la ocurrencia de eventos de precipitación modal, que de eventos extremos. Concluyendo, el avance de la antropización en la CARSCH generó una mayor producción de caudales ante eventos pluviales. La modelación hidrológica proporciónó información de la interacción entre el uso del suelo y el efecto de la erosividad pluvial con el fin de proyectar y pronosticar la influencia sobre la escorrentía superficial según el uso del suelo a nivel geoespacial.

Palabras clave: cambio de uso del suelo, simulación hidrológica, HEC-HMS, sierras bonaerenses.

\section{ABSTRACT}

Changes in coverage and land use are known as one of the main influences on surface water dynamics. Hydrological modeling is a tool for comparing rainfall and land use change scenarios in river basins. The purpose was to analyze the effect of land use and cover in the generation of runoff in the upper basin of Sauce Chico river 
(CARSCH), Argentina. A geospatial methodology of morphometric situation analysis was developed as a basic tool for hydrological modeling, which was carried out in HEC-HMS 4.2, applying the curve number (NC) model for an extreme rainfall event and another modal for comparison purposes of the response of the basin. The zoning of the vegetation cover was defined through a digital geoprocessing, for four scenarios of land use, considering them as representative of a trend of temporal space change. When analyzing the results according to rainfall events and land cover and use, it was determined that the highest drained volume and maximum flows in extreme storms is generated in mountain areas, while for modal events, the maximum is recorded in hill areas. In addition, the results reveal that the change in land use has a more significant influence on the behavior of flows in the event of modal precipitation events, rather than extreme events. In conclusion, the advance of the anthropization in the CARSCH generated a greater production of flows due to rain events. Hydrological modeling provided information on the interaction between land use and the effect of rainfall erosion in order to project and predict the influence on surface runoff according to land use at the geospatial level.

Keywords: land use change, hydrological simulation, HEC-HMS, Buenos Aires mountain ranges.

\section{INTRODUCCIÓN}

Los cambios de cobertura y uso del suelo son reconocidos como una de las principales causas de deterioro ambiental, con una influencia muy importante en la dinámica hídrica superficial. En este contexto, la cuenca hidrográfica es la unidad natural de tratamiento integrado y ámbito para la planificación y gestión del desarrollo sustentable, pues abarca todo el complejo biogeomórfico, económico y de relaciones sociales como unidad de planificación y gestión (Gaspari et al., 2009).

Los estudios sobre los procesos dinámicos de los cambios en la cobertura de suelo son importantes y necesarios, porque proporcionan la base para conocer las tendencias de los procesos de degradación, desertificación y pérdida de la biodiversidad de una región determinada (Van Lynden y Oldeman, 1997). 
En la zona serrana del sudeste de la provincia de Buenos Aires, la erosión hídrica superficial provoca la pérdida del horizonte superficial, que conlleva a una decreciente producción agropecuaria, que se ve contrarrestada con el actual manejo del suelo con la incipiente incorporación de medidas conservacionistas $y$, el avance de la tecnificación. En las últimas décadas, dada la capacidad productiva de los suelos de esta región, los pastizales pampeanos han sido sustituidos por agroecosistemas que evidencian un importante nivel de degradación y un escaso grado de conservación (Vázquez y Zulaica, 2011; Gaspari et al., 2019).

El cambio de uso del suelo genera una transformación importante en la dinámica del agua superficial y subterránea, debido al aumento del cubrimiento del suelo, eliminación de una cobertura vegetal absorbente, aumento de la extracción de agua de los acuíferos para riego, entre otros. Kaimowitz y Angelsen (1998) y Sandoval y Oyarzun (2003) han realizado estudios relacionados con el análisis y modelamiento del cambio en el uso del suelo, principalmente los referidos al proceso de deforestación en países tropicales.

El uso de modelos de proyección cartográfica del cambio en el uso del suelo es relativamente fiable, dada por la eficacia y exactitud espacial que le otorga el uso de bases de datos geográficos, integrados a un Sistemas de Información Geográfico (SIG) (Gaspari, 2007; Sandoval y Oyarzun, 2003; Rodríguez Vagaría et al., 2014). Estos modelos de cambio de uso se han transformado en una poderosa herramienta de análisis espacial orientada, principalmente, a los siguientes aspectos: (a) Explorar los variados mecanismos que fuerzan los cambios de uso del suelo y las variables sociales, económicas y espaciales que conducen a esto; (b) Proyectar los potenciales impactos ambientales y socioeconómicos derivados de los cambios en el uso del suelo, y (c) Evaluar la influencia de alternativas políticas y regímenes de manejo sobre los patrones de desarrollo y uso del suelo (Aguayo et al., 2007). La modelización hidrológica es una herramienta clave para comparar los escenarios de gestión y el cambio de uso del suelo en cuencas hidrográficas.

Según Senisterra et al. (2014) el conocimiento y evaluación de los cambios de uso del suelo y el efecto de la agresividad pluvial, es incipiente, especialmente cuando se analiza su integración al fenómeno precipitación-escorrentía. Numerosos trabajos demuestran la capacidad de distintos modelos hidrológicos para el análisis y la evaluación del comportamiento de una cuenca hídrica. Feleret al. (2014) mencionan 
que, en los últimos años, ha habido un desarrollo creciente y una mejora continua en el campo de la modelización hidrológica, que constituye una herramienta de interés para el análisis y evaluación de diferentes impactos en la disponibilidad y dinámica del recurso hídrico en cuencas hidrográficas.

El escurrimiento superficial es una de las variables hidrológicas más importante en el análisis de la dinámica hídrica y su modelización, considerado como el agua procedente de la lluvia que circula por la superficie y se concentra en los cauces. Su distribución temporal y espacial es función de las características topográficas, geológicas, climáticas y de vegetación de la cuenca y está íntimamente ligado a la relación entre aguas superficiales y subterráneas (Gaspari et al., 2009).

EL modelo hidrológico HEC-HMS $®$ (Hydrological Engineers Center-Hydrologic Modeling System) desarrollado del cuerpo de Ingenieros de los Estados Unidos (US Army Corp, 2017), permite simular el proceso de precipitación-escurrimiento en las distintas subcuencas que sean modeladas, sin importar el tamaño de las mismas. El mismo puede ser utilizado para distintos fines, como estudios de disponibilidad de agua, drenaje urbano, observaciones del flujo, impactos de intervenciones en las cuencas, o como en el presente estudio, para servir de herramienta de apoyo para realizar manejo de embalses (Bastidas, 2014). El programa cuenta con un sistema que integra diferentes métodos hidrológicos ya sean concentrados o distribuidos para tener como respuesta el tránsito de caudales. Esta característica del modelo permite flexibilidad combinando diferentes métodos de cálculo para cada parámetro que se desea obtener, logrando una mejor aproximación al sistema real (Tapia Aldas, 2012).

El objetivo fue analizar el efecto de la cobertura y uso del suelo sobre la generación de escorrentía ante un evento pluvial extremo en la cuenca alta de río Sauce Chico (CARSCH), Argentina.

\section{METODOLOGIA}

\section{Descripción del área de estudio}

El área de estudio comprende al sector serrano de la Cuenca del río Sauce Chico $\left(373,24 \mathrm{~km}^{2}\right)$, ubicada en la región pampeana en el suroeste de la Provincia de Buenos Aires (Figura 1). El curso principal nace en el cordón serrano BravardVentania con pendientes entre el 2-18\% (Gaspari, 2007). Se trata de una zona 
ganadera con agricultura importante para la provincia (Picardi \& Giacchero, 2015) y donde, a través del tiempo, las actividades agropecuarias sustituyen la vegetación natural por especies forrajeras, en las partes altas, y cultivos como trigo, avena, maíz, girasol, cebada y centeno, en las zonas de piedemonte y planicie. Sus principales características físicas están representadas por la topografía, influyendo en su dinámica biofísica, social y económica. El clima es templado y subhúmedo seco caracterizado por inviernos húmedos, veranos secos (Gaspari, 2007; Torrero, 2009).Los suelos presentes son del orden de los Molisoles, representados por asociaciones de: Argiudol típico en lomas, pendientes y planicies es muy profundo (140 cm); Haplustol típico, fino en el fondo del valle; Haplustoléntico y Haplustol lítico en los interfluvios, desarrollados sobre material loéssico que cubre totalmente las laderas de las sierras, y este a su vez se ubica sobre una costra calcárea (tosca) que oscila los $100 \mathrm{~cm}$ de espesor, según los diferentes accidentes que presente su topografía (FUNS, 2011; Gaspari, 2007).

La cuantificación de las variables morfométricas de $\mathrm{CARSCH}$, conformada por parámetros de forma, relieve y de drenaje, se implementó por medio de un análisis geoespacial de la elevación del terreno con el uso de técnicas de detección remota en sistema de información geográfica (SIG), mediante el procesamiento del modelo de elevación digital (MED - DEM por su siglas en inglés), SRTM (Shuttle Radar Topography Mission $)^{1}$ generado por la National Aeronautics and Space Administration (NASA) (Farr \& Kobrick, 2000). EI DEM se empleó para la delimitación de subcuencas y analizar el relieve conel programa QGIS 3.14 ( ), conformando la base de datos para la modelización hidrológica. Los parámetros morfométricos se expresan en la Tabla 1, según Guzmán Guaraca et al. (2021) y la Figura 2. Se identifica que las subcuencas 1 a 5 se desarrollan sobre un área serrana, la 6 en periserrana y las 7, 8 y 9 en zonas con lomadas (Gaspari et al., 2019).

${ }^{1} \mathrm{http}: / /$ srtm.csi.cgiar.org/srtmdata/ 


\section{revista estudios ambientales}

publicación digital del CINEA

Volumen $8 N^{\circ} 1$, año 2020

ISSN: 2347-0941

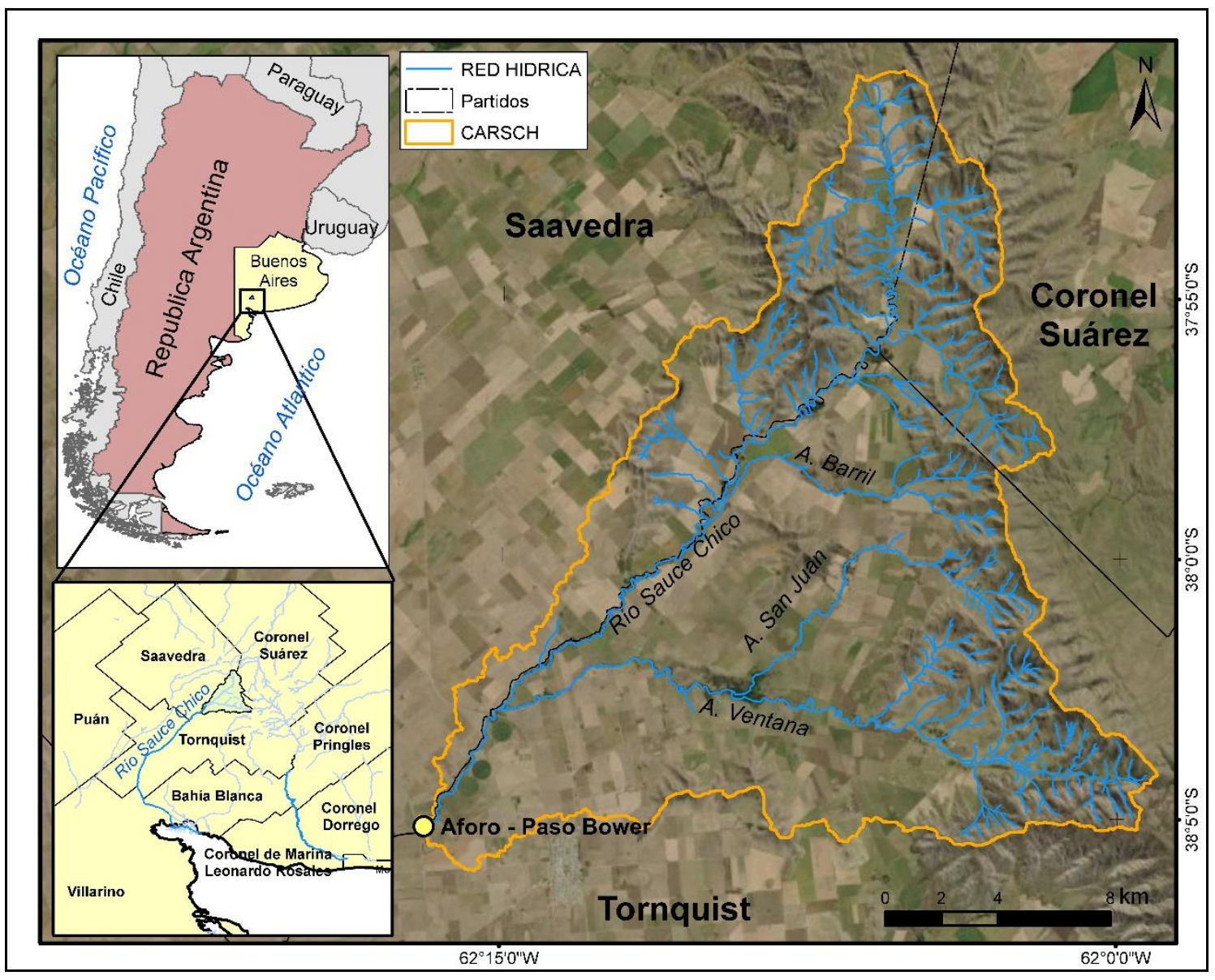

Figura 1. Ubicación de la cuenca alta del río Sauce Chico, Argentina

Tabla 1: Parámetros morfométricos de las unidades hidrológicas de $\mathrm{CARSCH}$

\begin{tabular}{|l|c|c|c|c|c|c|c|c|c|c|}
\hline \multicolumn{1}{|c|}{ Subcuenca } & $\mathbf{1}$ & $\mathbf{2}$ & $\mathbf{3}$ & $\mathbf{4}$ & $\mathbf{5}$ & $\mathbf{6}$ & $\mathbf{7}$ & $\mathbf{8}$ & $\mathbf{9}$ & CARSCH \\
\hline Parámetros & & & & & & & & & & \\
\hline Superficie $\left(\mathrm{km}^{2}\right)$ & 46,9 & 18,9 & 30,2 & 43,6 & 75,9 & 52,6 & 30,7 & 38,5 & 28,3 & 365,6 \\
\hline Perímetro (km) & 42,4 & 29,0 & 37,7 & 47,0 & 60,0 & 45,9 & 37,4 & 50,0 & 48,5 & 149,8 \\
\hline Longitud del cauce principal (km) & 14,5 & 8,8 & 14,9 & 15,8 & 18,0 & 17,4 & 12,5 & 13,8 & 7,0 & 52,7 \\
\hline Pendiente media del cauce principal $(\%)$ & 2,50 & 1,68 & 2,56 & 3,47 & 1,46 & 0,51 & 0,51 & 0,35 & 0,26 & 1,04 \\
\hline Altura máxima del cauce (m) & 800 & 551 & 540 & 512 & 508 & 414 & 335 & 321 & 278 & 800 \\
\hline Altura mínima del cauce (m) & 408 & 408 & 319 & 337 & 334 & 318 & 272 & 272 & 254 & 254 \\
\hline Orden de corrientes & 4 & 2 & 4 & 4 & 4 & 5 & 4 & 1 & 5 & 5 \\
\hline Densidad de drenaje (km/km²) & 1,95 & 1,85 & 1,10 & 0,82 & 1,36 & 1,46 & 0,44 & 0,41 & 0,25 & 1,13 \\
\hline Cantidad de cauces & 156 & 70 & 51 & 52 & 168 & 126 & 4 & 2 & 1 & 630 \\
\hline Densidad de corrientes (n-corrientes/km²) & 3,33 & 3,71 & 1,69 & 1,19 & 2,21 & 2,40 & 0,13 & 0,05 & 0,04 & 1,72 \\
\hline Índice de compacidad de Gravelius & 1,75 & 1,88 & 1,94 & 2,01 & 1,94 & 1,79 & 1,90 & 2,27 & 2,57 & 2,21 \\
\hline Coeficiente de rugosidad & 1,17 & 0,92 & 0,69 & 0,64 & 1,06 & 1,00 & 0,15 & 0,14 & 0,08 & 0,97 \\
\hline
\end{tabular}

Fuente: Guzmán Guaraca et al (2021).

El Tiempo de concentración (Tc) es un factor morfométrico que expresa el tiempo necesario para que todo el sistema contribuya eficazmente a la generación de flujo en 
el sitio de desagüe (Llamas, 1993) (en horas) que depende de las características geomorfológicas de la cuenca. Según Vélez y Botero (2010) para estimarlo pueden usarse diferentes fórmulas que se relacionan con parámetros propios de la cuenca, pero se recomienda emplear el valor medio obtenido de varias ecuaciones empíricas disponibles en la literatura científica (aplicando las ecuaciones de Wanielista et al., 1977; Clark, 1945; Giandotti, Arbeláez et al., 1997; Kirpich, 1940; Método Racional Generalizado, 1987;Pilgrim y McDermott, 1982; Témez, 1991 Valencia y Zuluaga; 1981), como es el caso de la aplicación en el presente trabajo en la CARSCH.

En cuanto al tiempo de rezago, se consideró la formulación de Vélez y Botero (2010) quienes afirman que la relación propuesta por el "Soil Conservation Service" (SCS)de Estados Unidos, es de 0,6 con respecto al tiempo de concentración medio estimado.

\section{Metodología aplicada}

El método del número de curva (NC) desarrollado por el SCS (1972) se basa en la estimación directa del escurrimiento superficial de una lluvia aislada para un complejo suelo - vegetación (Gaspari et al., 2015), en función del volumen y profundidad de la lámina generada (Wang et al., 2005; Rodríguez Vagaría y Gaspari, 2014). La estructura y textura del suelo y la cobertura vegetal junto a los registros pluviográficos, conforman los datos básicos para el análisis hidrológico espacio-temporal, en pequeñas cuencas hidrográficas (Gaspari et al; 2013).

El método de NC utiliza una ecuación para cuantificar el escurrimiento superficial $(\mathrm{Pe})$ que se expresa como el cociente entre $\left(P-l_{a}\right)^{2}$ y $\left(P-I_{a}+S\right)$, donde $P$ es la precipitación, $l_{a}$ es la abstracción inicial, $S$ es el máximo potencial de retención de la cuenca ante un evento de tormenta. El S se determina por el cociente entre (25.400 $254 \times \mathrm{NC}$ ), siendo el NC, representado por la combinación del grupo hidrológico de suelo, las clases de coberturas y usos del suelo y la condición de humedad antecedente del suelo (Gaspari et al., 2015).

La determinación del NC, se inició con la definición y zonificación del grupo hidrológico (GH) a nivel de subcuenca hidrográfica, el cual expresa la condición hidrológica del suelo según características texturales edáficas (SCS, 1964; Gaspari et al., 2013).A partir de la clase textural de los suelos según la clasificación de USDA (United States Departament of Agriculture, 1964; Rodríguez Vagaría et al., 2014) de la cartografía de 
suelos de INTA 1:50.000 (INTA, 2018), se establecieron los grupos hidrológicos (GH) en el área en estudio. Posteriormente, por medio del procesamiento e interpretación de los mapas de suelos, se generó el mapa de GH, con el programa QGis 3.14.

La zonificación de la cobertura vegetal se definió para cuatro escenarios de uso de suelo considerados como representativos de una tendencia de cambio espacio temporal (Tabla 1) desde un estado no antropizado a sobreexplotado por el hombre. Se desarrolló a través de un geoprocesamiento digital con el programa QGis 3.14, a partir de la interpretación de fotografías aéreas, imágenes satelitales y relevamiento de campo para los escenarios intermedios, en los años 1987 y 2017. El escenario del uso de suelo original (no antropizado) se estableció como una situación prístina. El escenario futuro, fue definido para una situación posible en el 2050, considerando una situación negativa, debido al aumento de la degradación por pérdida de suelo superficial.

Considerando la definición del $\mathrm{GH}$, y conociendo el tipo y distribución de la cobertura vegetal y/o el uso del suelo presente en cada subcuenca, para una condición de humedad antecedente media (II), aplicando un procesamiento geoespacial con SIG, se realizó una tabulación cruzada de la cartografía y con un Modelo Algebraico Cartográfico (Harvor, 2007; Gaspari et al, 2016) se zonificó el NC, cuyo valor se obtuvo de tablas.

La modelización se realizó aplicando dos tormentas obtenidas de la lectura de fajas pluviográficas, una extrema $(81,2 \mathrm{~mm}$ ) (año 1986) del Servicio Meteorológico Nacional de Tornquist y una modal (26,5 mm) (año 1990) de la Cooperativa Rural, ambas con una duración de 480 minutos, tomada cada 0,5 horas, siendo esta última considerada como testigo de las condiciones climáticas normales, representadas en la Figura 2. 


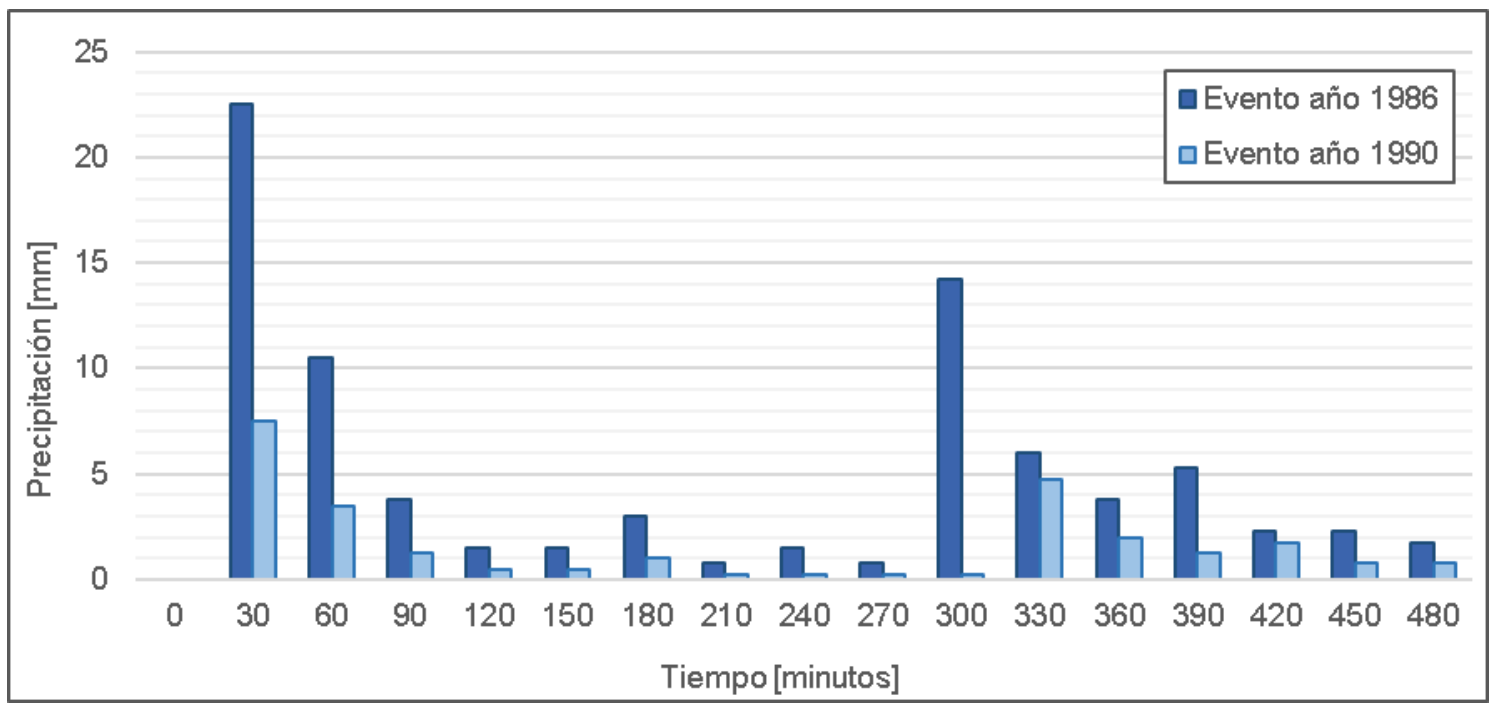

Figura 2. Tormentas empleadas en el análisis

La modelización hidrológica integradora se realizó con el programa HEC-HMS (2017) que presenta una interfaz gráfica que permite introducir la información necesaria para la simulación, manejar los componentes de análisis hidrológico y obtener respuestas en formato de gráfico o tablas. Los datos de entrada, en este caso de estudio, son el NC, cuya información que debe proveerse al modelo para realizar las simulaciones; el evento pluvial, la información morfométrica de las subcuencas datos tomados a campo para el proceso de transformación de lluvia en escorrentía, y las características hidráulicas de los tramos de canal y métodos de tránsito. La Figura 3 presenta la distribución de las unidades hidrológicas (subcuencas) de la CARSCH que representa el diagrama de secuencia de la dinámica hídrica superficial desde la cabecera hacia la desembocadura (Gaspari et al., 2019).

El punto de cierre de la CARSCH está dado por la estación de aforo Paso Bower, como se muestra en la Figura 1, que fue reactivada a partir del año 1997 con ciertos periodos de inactividad. El caudal promedio registrado por la Autoridad del Agua de la Provincia de Buenos Aires hasta el año 2018 es de 21,12 m³/s, el máximo valor fue de $101,51 \mathrm{~m}^{3} / \mathrm{s}$ y un mínimo de $1,06 \mathrm{~m}^{3} / \mathrm{s}$. Dado el carácter comparativo de los efectos derivados por las variaciones en la cobertura y uso del suelo sobre el escurrimiento superficial, se considera que no fueron afectados los resultados obtenidos en las modelaciones por la ausencia de mediciones de aforo. 


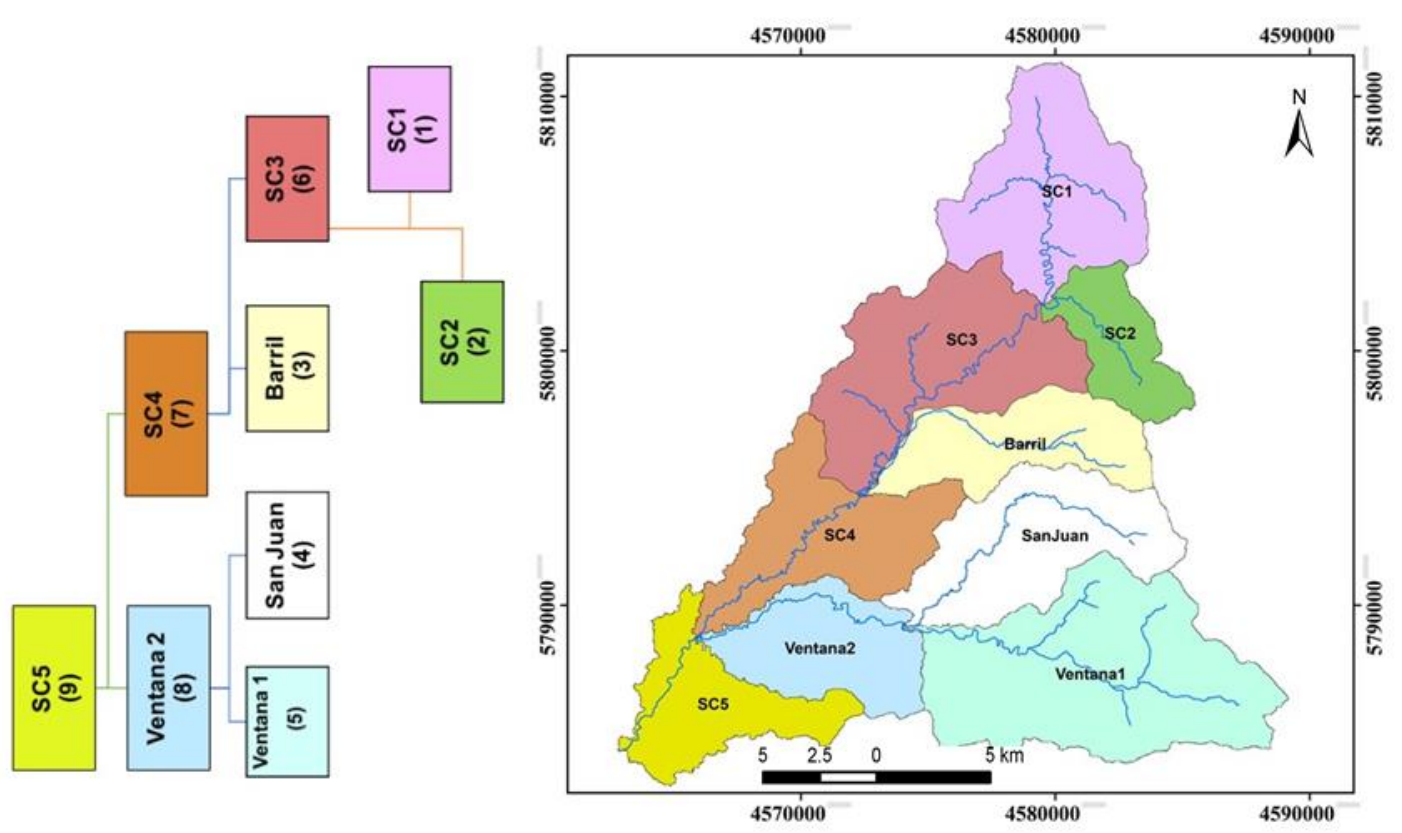

Figura 3. Distribución de las unidades hidrológicas (subcuencas) y su diagrama de bloques Fuente: Gaspari et al., 2019.

El análisis del efecto de la cobertura y uso del suelo sobre la generación de escorrentía en la CARSCH se realizó en ocho modelaciones (I a VIII) donde se ensayaron los cuatro escenarios de uso del suelo, como se presenta en la Tabla 2 y, en cada uno de ellos, se aplicaron las tormentas mencionadas: un evento pluvial extremo y uno modal.

Tabla 2. Escenarios de modelación hidrológica

\begin{tabular}{|c|c|c|}
\hline Precipitación & Escenario de uso de suelo & Modelación \\
\hline \multirow{4}{*}{$81,2 \mathrm{~mm}$} & Original & $\mathrm{I}$ \\
\cline { 2 - 3 } & 1987 & $\mathrm{II}$ \\
\cline { 2 - 3 } & 2017 & $\mathrm{III}$ \\
\cline { 2 - 3 } & Futuro & $\mathrm{IV}$ \\
\hline \multirow{3}{*}{$26,5 \mathrm{~mm}$} & Original & $\mathrm{V}$ \\
\cline { 2 - 3 } & 1987 & $\mathrm{VI}$ \\
\cline { 2 - 3 } & 2017 & $\mathrm{VII}$ \\
\cline { 2 - 3 } & Futuro & $\mathrm{VIII}$ \\
\hline
\end{tabular}




\section{RESULTADOS Y DISCUSIÓN}

A partir de los datos de la Tabla 1, se calculó el tiempo de concentración a nivel de subcuenca, obteniendo la Tabla 3, estableciendo el valor promedio en cada caso, para utilizar en la modelización hidrológica de los cuatro escenarios de uso del suelo, según la metodología propuesta por Vélez y Botero (2010).

Tabla 3. Tiempo de concentración y rezago

\begin{tabular}{|l|c|c|c|c|c|c|c|c|c|c|}
\hline $\begin{array}{c}\text { Tiempo de concentración } \\
\text { (hrs) }\end{array}$ & $\mathbf{1}$ & $\mathbf{2}$ & $\mathbf{3}$ & $\mathbf{4}$ & $\mathbf{5}$ & $\mathbf{6}$ & $\mathbf{7}$ & $\mathbf{8}$ & $\mathbf{9}$ & CARSCH \\
\hline Bransby-Williams & 1,99 & 1,43 & 2,14 & 2,05 & 2,63 & 3,26 & 2,47 & 2,89 & 1,60 & 7,05 \\
\hline California Culvert Practice & 2,08 & 1,72 & 2,70 & 3,14 & 3,66 & 4,42 & 3,55 & 4,41 & 2,64 & 8,16 \\
\hline Clark & 2,50 & 1,64 & 1,91 & 2,17 & 3,91 & 4,30 & 3,11 & 4,00 & 3,64 & 10,97 \\
\hline Giandotti & 3,22 & 3,15 & 2,84 & 2,67 & 4,77 & 7,33 & 6,39 & 8,23 & 9,38 & 8,32 \\
\hline Kirpich & 2,14 & 1,70 & 2,18 & 2,02 & 3,12 & 4,56 & 3,53 & 4,44 & 2,95 & 8,15 \\
\hline Método Racional Generalizado & 2,83 & 1,82 & 2,03 & 2,13 & 4,06 & 4,43 & 0,52 & 0,62 & 0,21 & 7,69 \\
\hline Pilgrim y McDermott & 3,28 & 2,32 & 2,77 & 3,19 & 3,94 & 3,43 & 2,79 & 3,04 & 2,71 & 7,16 \\
\hline Témez & 1,92 & 1,42 & 1,96 & 1,93 & 2,51 & 2,99 & 2,32 & 2,70 & 1,70 & 6,06 \\
\hline Valencia y Zuluaga & 3,66 & 3,21 & 3,15 & 3,23 & 4,91 & 5,94 & 5,13 & 6,12 & 6,46 & 8,15 \\
\hline \multicolumn{1}{|c|}{ Promedio } & $\mathbf{2 , 6 3}$ & $\mathbf{2 , 0 5}$ & $\mathbf{2 , 4 1}$ & $\mathbf{2 , 5 0}$ & $\mathbf{3 , 7 2}$ & $\mathbf{4 , 5 2}$ & $\mathbf{3 , 3 1}$ & $\mathbf{4 , 0 5}$ & $\mathbf{3 , 4 8}$ & $\mathbf{7 , 9 7}$ \\
\hline \multicolumn{1}{|c|}{ Tiempo de Rezago } & $\mathbf{1 , 5 8}$ & $\mathbf{1 , 2 3}$ & $\mathbf{1 , 4 5}$ & $\mathbf{1 , 5 0}$ & $\mathbf{2 , 2 3}$ & $\mathbf{2 , 7 1}$ & $\mathbf{1 , 9 9}$ & $\mathbf{2 , 4 3}$ & $\mathbf{2 , 0 9}$ & $\mathbf{4 , 7 8}$ \\
\hline
\end{tabular}

Los cuatro escenarios espacio temporales de cobertura y usos del suelo planteados para la modelización hidrológica en la CARSCH responden a la descripción dela Tabla 4. Se trabajó en base a dichas estructuras de cobertura vegetal, debido a que el cambio de uso del suelo en la cuenca manifestó una variación en las características de tipo y estado de cobertura y porcentajes de recubrimiento de la vegetación. 
Tabla 4. Distribución porcentual en superficie de usos del suelo, en CARSCH

\begin{tabular}{|c|c|c|c|c|}
\hline \multicolumn{4}{|c|}{ Escenarios de cobertura y uso del suelo* } & \multirow{2}{*}{$\begin{array}{c}\text { \% de } \\
\text { ocupación en } \\
\text { CARSCH }\end{array}$} \\
\hline Original & 1987 & 2017 & Futura & \\
\hline \multirow{6}{*}{$\begin{array}{c}\text { Pastizal } \\
\text { mezclado } \\
\text { con pajonal } \\
\text { con la mejor } \\
\text { cobertura y } \\
\text { CHB }\end{array}$} & $\begin{array}{c}\text { Cultivos en } \\
\text { hileras rectas - } \\
\text { CHB }\end{array}$ & $\begin{array}{c}\text { Cultivos no } \\
\text { alineados o } \\
\text { surcos pequeños } \\
\text { - CHB }\end{array}$ & $\begin{array}{c}\text { Cultivo en hileras - } \\
\text { CHP }\end{array}$ & $49 \%$ \\
\hline & $\begin{array}{l}\text { Montes con } \\
\text { pastos - CHP }\end{array}$ & $\begin{array}{l}\text { Montes con } \\
\text { pastos- CHP }\end{array}$ & \begin{tabular}{|c|} 
Bosque con \\
mezcla de pastos \\
- CHP \\
\end{tabular} & $1 \%$ \\
\hline & $\begin{array}{c}\text { Prados } \\
\text { permanentes }\end{array}$ & Pastizal - CHB & $\begin{array}{c}\text { Pastizal medio } \\
\text { CHP }\end{array}$ & $14 \%$ \\
\hline & $\begin{array}{c}\text { Matorral, mezcla } \\
\text { matorral y } \\
\text { maleza mejor } \\
\text { cubierta }>75 \%\end{array}$ & $\begin{array}{c}\text { Matorral, mezcla } \\
\text { matorral y } \\
\text { maleza cubierta } \\
50 \%\end{array}$ & $\begin{array}{c}\text { Mezcla matorral y } \\
\text { malezas }- \\
\text { cobertura }<50 \%\end{array}$ & $3 \%$ \\
\hline & \multirow{2}{*}{ Pastizal - CHP } & Pastizal - CHP & $\begin{array}{c}\text { Pastizal con roca } \\
\text { en superficie, } \\
\text { suelo poco } \\
\text { profundo - CHP }\end{array}$ & $32 \%$ \\
\hline & & $\begin{array}{l}\text { Residencial de } \\
\text { baja densidad }\end{array}$ & $\begin{array}{c}\text { Granjas, } \\
\text { construcciones, } \\
\text { caminos y lotes } \\
\text { circundantes }\end{array}$ & $1 \%$ \\
\hline
\end{tabular}

${ }^{*}$ CHP: Condición Hidrológica Pobre -- CHB: Condición Hidrológica Buena

La zonificación de cobertura y uso del suelo definidos para el año 2017, determinó que el proceso de urbanización (residencial de baja densidad) y el desarrollo de la actividad agropecuaria han generado la pérdida de calidad en los pastizales nativos, que propició la disminución de la protección por cobertura debido a la pobre recuperación natural. El uso agropecuario presentó una escasa incorporación de medidas de conservación de suelos y el monte con forestaciones expresó de mayor cobertura foliar, no así extensión.

El análisis textural de los suelos presentes en la CARSCH permitió zonificar los GH cuya ocupación en superficie se exponen en la Tabla 5, siendo el $D$ el de menor proporción en la cuenca y el B se presenta desde la cabecera hacia la zona periserrana. 
Tabla 5. Superficie de ocupación porcentual del GH por subcuenca en la CARSCH

\begin{tabular}{|c|l|c|c|c|}
\hline \multirow{2}{*}{ Subcuenca } & \multicolumn{3}{c|}{ Superficie porcentual según GH } \\
\cline { 3 - 5 } & B & C & D \\
\hline $\mathbf{1}$ & SC1 & 87,52 & 12,48 & 0,00 \\
\hline $\mathbf{2}$ & SC2 & 93,54 & 6,46 & 0,00 \\
\hline $\mathbf{3}$ & Barril & 92,18 & 7,82 & 0,00 \\
\hline $\mathbf{4}$ & San Juan & 69,26 & 29,96 & 0,78 \\
\hline $\mathbf{5}$ & Ventana 1 & 51,13 & 48,87 & 0,00 \\
\hline $\mathbf{6}$ & SC3 & 85,44 & 14,56 & 0,00 \\
\hline $\mathbf{7}$ & SC4 & 82,06 & 15,68 & 2,26 \\
\hline $\mathbf{8}$ & Ventana 2 & 23,56 & 76,44 & 0,00 \\
\hline $\mathbf{9}$ & SC5 & 21,62 & 76,90 & 1,48 \\
\hline \multicolumn{2}{|c|}{ CARSCH } & 67,13 & 32,43 & 0,45 \\
\hline
\end{tabular}

La zonificación de los cuatro escenarios de cobertura y uso del suelo (Tabla 4) se integró con los grupos hidrológicos del suelo (Tabla 5) para establecer la distribución geoespacial del NC dentro de cada subcuenca, como se muestra en la Figura 4. 


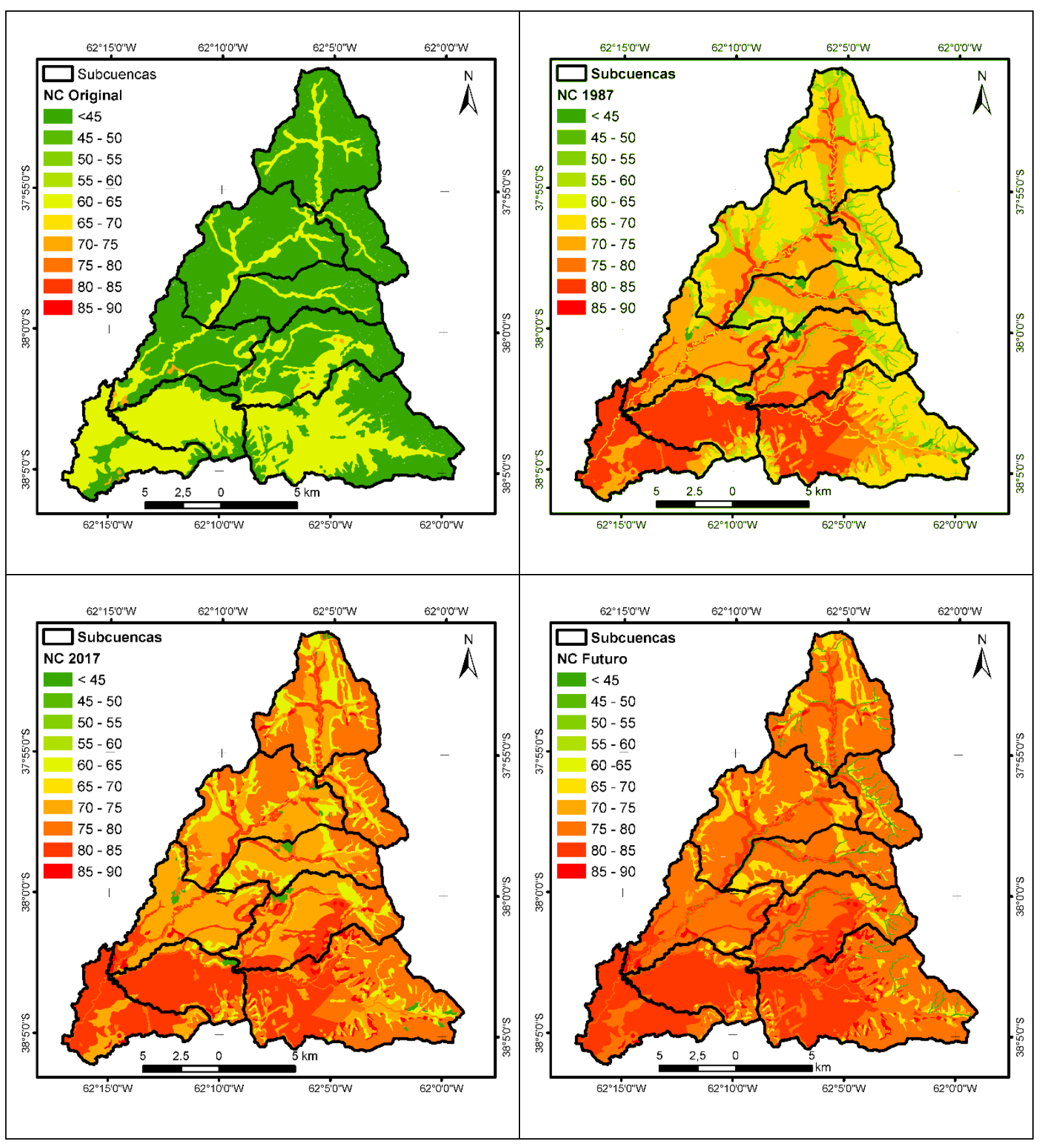

Figura 4. Distribución del NC según escenarios de cobertura y uso del suelo.

A partir del análisis de la zonificación del NC para los cuatro escenarios, se definió el NC ponderado por subcuenca, como se expresa la Tabla 6. 
En la Tabla 6 se evidencia que, para los cuatro escenarios, los NC de las subcuencas 1 a 5, ubicadas en el área serrana, son menores a los determinados para las subcuencas del 6 al 9, en el área periserrana a lomadas. Esto se debe, principalmente, al tipo y estructura de la cobertura vegetal presente. Asimismo, refleja el aumento en el NC para una misma subcuenca a través del tiempo (escenarios), como consecuencia de la degradación del suelo debido a cambios en su uso.

Tabla 6. Resumen de los NC ponderados por escenario

\begin{tabular}{|c|l|c|c|c|c|}
\hline \multirow{2}{*}{ Subcuenca } & \multicolumn{4}{|c|}{ NC ponderado por escenario } \\
\cline { 3 - 6 } & Original & $\mathbf{1 9 8 7}$ & $\mathbf{2 0 1 7}$ & Futuro \\
\hline 1 & SC1 & 50,12 & 66,31 & 74,41 & 76,95 \\
\hline 2 & SC2 & 49,08 & 65,70 & 75,84 & 77,30 \\
\hline 3 & Barril & 49,32 & 67,47 & 73,78 & 77,19 \\
\hline 4 & San Juan & 53,29 & 71,62 & 76,62 & 79,40 \\
\hline 5 & Ventana 1 & 56,31 & 71,34 & 78,26 & 79,99 \\
\hline 6 & SC3 & 50,48 & 68,41 & 74,93 & 77,85 \\
\hline 7 & SC4 & 51,23 & 71,83 & 75,64 & 79,15 \\
\hline 8 & Ventana 2 & 61,00 & 76,80 & 80,27 & 82,05 \\
\hline 9 & SC5 & 61,44 & 77,96 & 80,78 & 82,47 \\
\hline
\end{tabular}

Los datos morfométricos de la Tabla1, los tiempos de concentración y rezago de la Tabla 3y los NC correspondientes por subcuenca de la Tabla 6, se integraron para la modelización en la CARSCH con el HEC HMS, para los ocho escenarios planteadas en la Tabla 2. El esquema de la distribución de las unidades hidrológicas para la secuencia de la modelización hidrológica se presenta en la Figura 5.

Los resultados alcanzados de la modelación del escurrimiento superficial para las ocho situaciones se presentan en la Tabla 7, los cuales proporcionan información sobre la dinámica hídrica superficial como consecuencia de los cambios espacio-temporales de uso del suelo en la CARSCH. En relación con las modelaciones realizadas con la tormenta extrema, la respuesta en las subcuencas del área serrana fue mayor, a excepción de SC2 dada su menor área de aporte. Hacia la desembocadura, en el área de lomadas, Ventana 2 y SC4, dicha respuesta está contenida por la disminución de las pendientes. Mientras que, en las modelaciones realizadas para la tormenta modal, la respuesta de las subcuencas en la zona periserrana y de lomadas fue mayor 
generando un caudal pico superior, quedando contenida el agua por infiltración en las cabeceras donde los NC son menores.

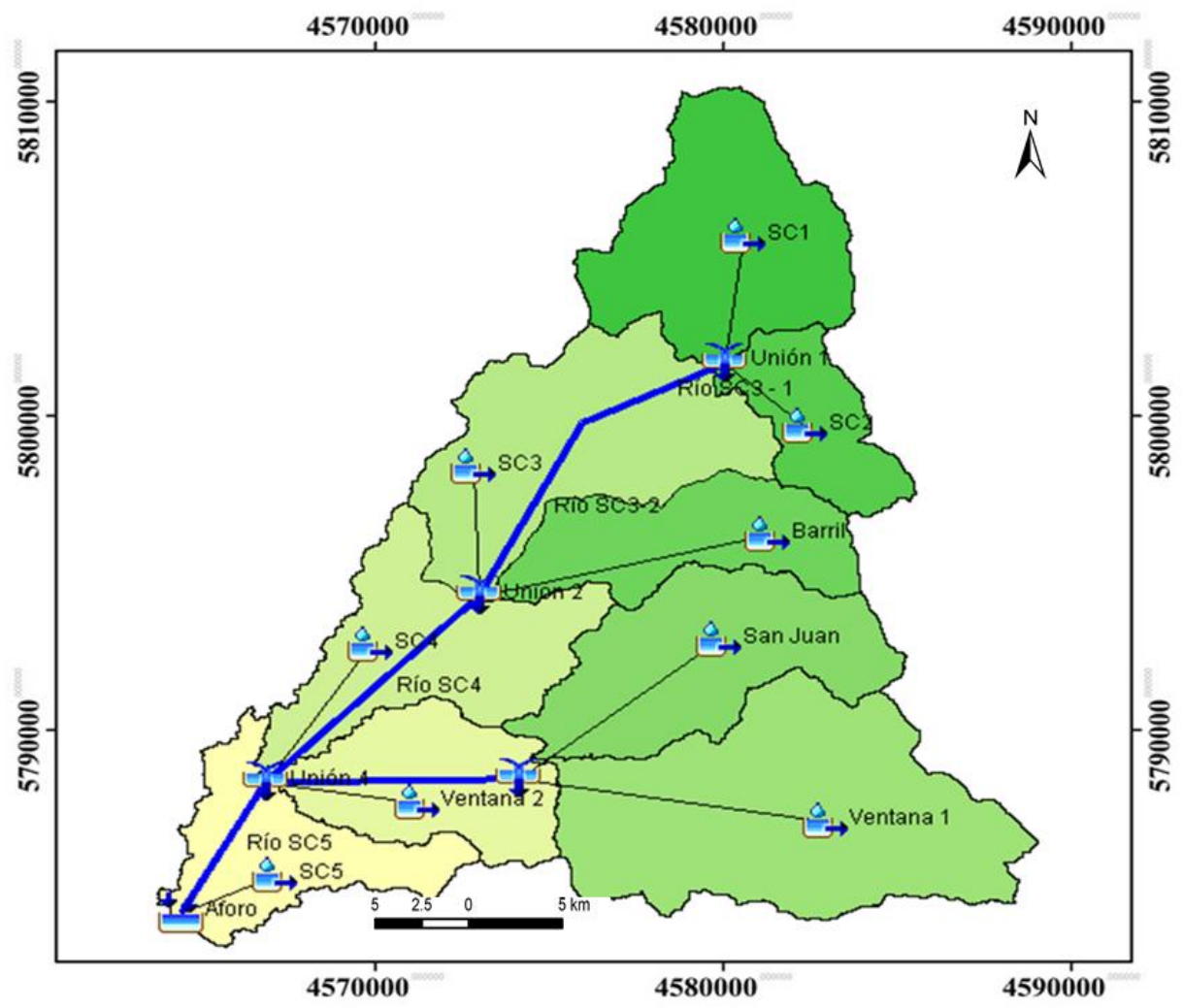

Figura 5. Esquema de la modelación de las unidades hidrológicas de la CARSCH para HECHMS 4.2.1

En la Tabla 7 se presentan los resultados para las modelizaciones hidrológicas para los cuatro escenarios, donde se observa que el volumen de escurrimiento responde espacialmente a la naturaleza del complejo suelo-vegetación, su estado y su morfometría. Los mayores valores de escurrimiento se presentaron en las modelaciones I a IV, en las áreas de pastizales coincidentes con en las subcuencas serranas con elevadas pendientes (superior a $40 \%$ ) y suelos rocosos de poco desarrollo (subcuencas 1 a 5), que son los que originan el mayor aporte hídrico en cuanto se degrada la condición de cobertura vegetal a través del tiempo, forjando una tendencia positiva de escurrimiento desde I hacia IV. En los casos de aplicación de una tormenta modal (V a VIII) se visualiza que para la modelación V la lluvia es retenida completamente en toda la cuenca. Mientras que para la modelación $\mathrm{VI}$, se genera los mayores escurrimientos en las subcuencas de la desembocadura. 
Finalmente, en las modelaciones VII y VIII, toda la $\mathrm{CARSCH}$ genera escurrimiento en forma proporcional a su morfometría y a la evolución del complejo suelo-vegetación.

Tabla 7. Escurrimiento porcentual por subcuenca según modelación*

\begin{tabular}{|l|c|c|c|c|c|c|c|c|}
\hline \multirow{2}{*}{$\begin{array}{c}\text { Elemento } \\
\text { hidrológico }\end{array}$} & \multicolumn{7}{|c|}{ Volumen escurrido $\left(\mathbf{1 0 0 0} \mathbf{~ m}^{\mathbf{3}}\right.$ ) } \\
\cline { 2 - 9 } & $\mathbf{I}$ & $\mathbf{I I}$ & $\mathbf{I I I}$ & $\mathbf{I V}$ & $\mathbf{V}$ & $\mathbf{V I}$ & VII & VIII \\
\hline SC1 (1) & 155,3 & 779,7 & 1260 & 1436,4 & 0 & 0,2 & 39,6 & 68,3 \\
\hline SC2 (2) & 52,5 & 301,6 & 547,2 & 589,1 & 0 & 0 & 22 & 29,4 \\
\hline SC3 (6) & 184,4 & 1001,4 & 1452,5 & 1684,7 & 0 & 0,2 & 50,2 & 90,5 \\
\hline Barril (3) & 87,5 & 541,7 & 784,4 & 936,1 & 0 & 4 & 21,8 & 46 \\
\hline Ventana 1 (5) & 555,2 & 1723,7 & 2483,4 & 2698,5 & 0 & 26,1 & 140,5 & 186,8 \\
\hline San Juan (4) & 226,1 & 1005,3 & 1204,4 & 1506 & 0 & 16,5 & 59,6 & 97,6 \\
\hline SC4 (7) & 151,3 & 899,2 & 1104,7 & 1315,3 & 0 & 15,7 & 43,1 & 82,9 \\
\hline Ventana 2 (8) & 345,5 & 934,7 & 1106,4 & 1201,3 & 0 & 43,5 & 78,9 & 102,5 \\
\hline SC5 (9) & 329,9 & 912,2 & 1043,8 & 1128 & 0 & 49,7 & 78,6 & 100,2 \\
\hline
\end{tabular}

*Azul: Tormenta extrema, Verde Tormenta modal.

La Tabla 8 presenta los caudales emitidos a la salida de cada subcuenca para las ocho modelaciones, es importante referirse a la extrema relación de la morfometría de cada unidad hidrológica con la generación de caudales, donde tanto su superficie como su relieve generan indicadores hídricos básicos que se reconocen en este análisis de la dinámica superficial, como se expresa en las subcuencas SC1, Ventana 1 y San Juan.

Tabla 8. Caudales de la modelación según evento pluvial y condición de uso del suelo

\begin{tabular}{|l|c|c|c|c|c|c|c|c|}
\hline \multirow{2}{*}{$\begin{array}{c}\text { Elemento } \\
\text { hidrológico }\end{array}$} & \multicolumn{7}{|c|}{ Caudal pico por modelación $\left.\mathbf{( m}^{\mathbf{3}} / \mathbf{s}\right)$} \\
\cline { 2 - 9 } & $\mathbf{I}$ & II & III & IV & V & VI & VII & VIII \\
\hline SC1 (1) & 12,2 & 52 & 76,4 & 84,4 & 0 & 0 & 3,5 & 5,7 \\
\hline SC2 (2) & 4,5 & 21,6 & 35,1 & 37,2 & 0 & 0 & 2 & 2,6 \\
\hline SC3 (6) & 11,5 & 52,9 & 71,4 & 80,1 & 0 & 0 & 3,3 & 5,9 \\
\hline Barril (3) & 7,1 & 36 & 48,1 & 55,3 & 0 & 0,3 & 2 & 3,9 \\
\hline Ventana 1 (5) & 36,4 & 95,8 & 127,5 & 135,7 & 0 & 2,1 & 10 & 12,9 \\
\hline San Juan (4) & 17 & 62,9 & 72,4 & 85,6 & 0 & 1,6 & 5 & 7,7 \\
\hline SC4 (7) & 9,4 & 45,9 & 54,1 & 61,8 & 0 & 1,1 & 2,9 & 5,3 \\
\hline Ventana 2 (8) & 21,5 & 49,2 & 55,9 & 59,3 & 0 & 3,2 & 5,4 & 6,8 \\
\hline SC5 (9) & 20,2 & 46,1 & 51 & 54 & 0 & 3,5 & 5,3 & 6,5 \\
\hline Aforo & 87,1 & 383,5 & 515,6 & 576,6 & 0 & 5,1 & 19,8 & 29,7 \\
\hline
\end{tabular}


Según Henríquez et al. (2006) y Rodríguez Vagaría et al. (2014), si se reduce la cobertura de árboles y vegetación, decrece la evapotranspiración de 40\% a 25\%; aumenta la tasa de escorrentía de $10 \%$ a 30\%; disminuye el tiempo de rezago entre la iniciación de precipitaciones y escorrentía; y decrece la infiltración subterránea de 50\% a $32 \%$. En coincidencia con estos antecedentes, en el caso de la CARSCH se afirma que uno de los más notables impactos ambientales del reemplazo de coberturas naturales por coberturas semi-impermeables, se corresponde con el aumento de la escorrentía superficial.

En coincidencia con Mármol (2006) y Díaz Gómez \& Gaspari (2017), los cambios de uso y cobertura del suelo presentan una compleja relación con la dinámica hídrica en una cuenca hidrográfica, ya que influye en la intercepción de la lluvia, retardo de la escorrentía y aumento de la infiltración, regulando de este modo el rendimiento hídrico de la cuenca y evitando crecientes e inundaciones, causando impactos tanto en el comportamiento hidráulico como hidrológico.

\section{CONCLUSIONES}

Este trabajo permitió analizar el cambio de uso del suelo ante un evento extremo de precipitación y su comparación con uno modal, proporcionando información de la interacción entre la cobertura vegetal y el efecto de tormentas de igual duración y distinta intensidad. Esta interacción permite proyectar y pronosticar la influencia sobre la escorrentía superficial según el uso del suelo, a nivel geoespacial.

El análisis de tendencia espacio temporal de la cobertura y uso del suelo vislumbró que los cambios reflejaron un aumento del escurrimiento, debido a la desprotección y reducción de cubrimiento por la vegetación, en la tormenta modal. En el escenario pluvial extremo, la morfometría de la cuenca fue determinante sobre la respuesta del escurrimiento y generación de caudales.

Al analizar la cobertura y uso del suelo, en los cuatro escenarios, sobre el efecto de las diferencias de caudales según los eventos pluviales, se determinó que el mayor volumen escurrido y los caudales máximos en la tormenta extrema se genera en áreas serranas en la CARSCH, mientras que ante eventos modales los máximos se registran en zonas de lomadas. Además, los resultados revelan que el cambio en el uso del suelo tiene una mayor influencia en el comportamiento de los caudales ante la 
ocurrencia de eventos de precipitación modal, que de eventos extremos. Es importante concluir que el avance de la antropización en la CARSCH generó una mayor producción de caudales antes eventos pluviales.

\section{AGRADECIMIENTOS}

La financiación de este trabajo fue desarrollada por el Proyecto denominado "Estudio de los servicios hidrológico-ambientales para aportar a la sustentabilidad de la Región Serrana Bonaerense", del Programa de Incentivos a los Docentes - Investigadores Secretaria de Política Universitaria - Ministerio de Educación de la Nación. Proyectos I+D 2016. Director: Dra. Fernanda Gaspari. Acreditado. Proy. № 11/A286. 2016-2019.

\section{BIBLIOGRAFIA}

Aguayo, M. I., T. Wiegand, G. D. Azócar, K. Wiegand \& Vega, C. E. (2007). Revealing the driving forces of mid-cities urban growth patterns using spatialmodeling: a case study of Los Angeles, Chile. Ecology and Society 12 (1): 13. Disponible en: http:// www. ecologyandsociety.org/ vol12/iss1/art13/.

Bastidas, C. (2014). Manual para el uso del modelo hidrológico HEC-HMS. Venezuela. Disponible en: https://www.academia.edu/36127656/Manual_para_el_uso_del_ modelo_hidrol\%C3\%B3gico_HEC-HMS

Díaz Gómez, A.R. \& Gaspari, F. J. (2017). Modelización geoespacial del escurrimiento superficial en cuencas vulnerables al cambio de uso del suelo. Revista CINEA. Volumen $5 \mathrm{~N}^{\circ}$ 1: 29-47. Disponible en: http://ojs.fch.unicen.edu.ar/index.php/ estudios-ambientales/article/view/143/122

Farr, T.G., \& Kobrick, M. (2000). Shuttle Radar Topography Mission produces a wealth of data, Amer. Geophys. Union Eos, v. 81, p. 583-585.

Feler, M.V.; Vazquez-Amabile, G.; van Meer, H.; Gaspari, F.J.; Mercuri, P.A.; Prieto, D.; \&Flamenco, E. (2014). Validación del modelo SWAT en la cuenca del río Quequén Grande y evaluación de potenciales cambios a nivel de cuenca. $2^{\circ}$ Congreso Internacional de Hidrología de Llanuras. Santa Fe, Argentina.

Fundación de la Universidad Nacional del Sur FUNS (2011). Prefactibilidad e sistematización y aprovechamiento de la cuenca del Río Sauce Chico. FUNS. 
Disponible en: http://biblioteca.cfi.org.ar/documento/prefactibilidad-de-sistematizaci on-y-aprovechamiento-de-la-cuenca-del-rio-sauce-chico-provincia-de-buenosaires/

Gaspari, F J. (2007). Ordenamiento territorial en cuencas serranas aplicación de sistemas de información geográfica (SIG), Ediciones Cooperativas. 1a edición. Buenos Aires. Argentina. 10,116 pp.

Gaspari, F.J., Senisterra, G.E., Delgado, M.I., Rodríguez Vagaría, A. \& Besteiro, S. (2009). Manual de Manejo Integral de Cuencas Hidrográficas. Primera Edición. La Plata. $321 \mathrm{pp}$.

Gaspari, F., Rodríguez Vagaría, A., Senisterra, G., Delgado, M. I., \& Besteiro, S. (2013). Elementos metodológicos para el Manejo de Cuencas Hidrográficas. Curso de Manejo de Cuencas Hidrográficas. Editorial de la Universidad Nacional de La Plata. 188 p. La Plata. Argentina. Junio de 2013. Actualización permanente SEDICI.ISBN 978-950-34-0963-3. Versión digital. Disponible en http://sedici.unlp.edu.ar/handle/10915/27877

Gaspari, F. J., Senisterra, G. E. \& Díaz Gómez, A. R. (2015). Modelización hidrológica con L-THIA en la Cuenca Alta del Arroyo Napaleofú, Buenos Aires, Argentina. XXV Congreso Nacional del Agua. Paraná. Argentina. 8 p.

Gaspari, F., Senisterra, G., Delgado, M. I., Denegrí, G.A., Rodríguez Vagaría, A. \& Díaz Gómez R. (2016). Valoración de servicios ambientales para el ordenamiento agrohidrológico en cuencas hidrográficas. Editorial de la Universidad Nacional de La Plata. 137 p. La Plata. Argentina. SEDICI. ISBN 978-950-34-1324-1. Versión digital. Disponible en http://sedici.unlp.edu.ar/handle/10915/52722

Gaspari, F., de Antueno, L.; Guzmán Guaraca, A.; Senisterra, G.; Montealegre Medina F. \& Díaz Gómez, A. R. (2019). Modelización hidrológica en la cuenca alta del Arroyo Sauce Chico, Buenos Aires, Argentina. I Congreso Latinoamericano y V Nacional de Manejo de Cuencas Hidrográficas 2019. México. http://remexcu.org/cmch-2019/images/docs/Programa_CMCH-2019_V5.pdf

Guzmán Guaraca, A., de Antueno, L. \& Gaspari, F. (2021). Análisis de la variabilidad geoespacial de la fragilidad morfométrica en la cuenca alta del Río Sauce Chico, Argentina. Revista de la Facultad de Agronomía. La Plata Volumen 120. Número 1 de 2021. En edición. 
Harbor, J. M. (2007). A Practical Method for Estimating the Impact of Land-Use Change on Surface Runoff, Groundwater Recharge and Wetland Hydrology, Journal of the American Planning Association, 60:1, 95-108, DOI: $10.1080 / 01944369408975555$

Henríquez, C.; Azocar, G. \& Aguayo, M. (2006). Cambio de uso del suelo y escorrentía superficial: aplicación de un modelo de simulación espacial en Los Ángeles, VIII Región del Biobío, Chile. Revista de Geografía Norte Grande, Santiago de Chile, n.36. Disponible en: http://www.scielo.cl/scielo.php?script=sci_arttext\&pid=S0718$34022006000200004 \& \operatorname{lng}=e s \& n r m=i s o$

Instituto Nacional de Tecnología Agropecuaria INTA. (2018). Cartas de suelos de la Provincia de Buenos Aires. Disponible en: http://anterior.inta.gov.ar/suelos/ cartas/index.htm

Kaimowitz, D. \& Angelsen, A. (1998). Economic Models of Tropical deforestation. A Review, Center for International Forestry Research, Malaysia 305 pp.

Llamas, J. (1993). Hidrología general: Principios y Aplicaciones. Servicio Editorial de la Universidad del País Vasco. pp 635.

Mármol, L. A. (2006). Introducción al Manejo de Cuencas Hidrográficas y Corrección de Torrentes. Universidad Nacional de Salta. Facultad de Ciencias Naturales. Ingeniería en Recursos Naturales y Medio Ambiente. 287 pp.

Picardi, M. \& Giacchero, A. (2015). Productividad de la tierra agrícola en el sudoeste bonaerense. Estudios Económicos, 32. Disponible en: http://bibliotecadigital.uns. edu.ar/scielo.php?script=sci_arttext\&pid=S252512952015002200004\&lng=en\&nrm =iso

Rodríguez Vagaría, A.M. \& Gaspari, F. J. (2014). Modelización espacial del escurrimiento medio anual en la cuenca serrana bonaerense. Séptimo Congreso de la Ciencia Cartográfica "Logros y desafíos de la Cartografía". Buenos Aires. Argentina.

Sandoval, V. \& Oyarzun, V. (2003). Modelamiento y prognosis espacial del cambio en el uso del suelo. Quebracho.11. Santiago del Estero. Argentina. 9-21 pp.

Soil Conservation Service (SCS). (1964). Hydrology. Part I. Wathershed Planning. S.C.S. National Engineering Handbook. Section 4. U.S. Department of Agriculture. Washington. 33 pp. 
Senisterra, G.E., Gaspari, F.J. \& Mazzucchelli, M.G. (2014). Análisis espacio temporal del cambio de uso del suelo en una cuenca serrana de la provincia de Buenos Aires, Argentina. Séptimo Congreso de la Ciencia Cartográfica "Logros y desafíos de la Cartografía". Buenos Aires. Argentina.

Tapia Aldas, J.C. (2012). Modelización hidrológica de un área experimental en la cuenca del Río Guayas en la producción de caudales y sedimentos. Ecuador. Tesis de Magíster Scientiae en Manejo Integral de Cuencas Hidrográficas. F.C.A.F. - U.N.L.P. 239 pp.

Torrero, M. (2009). Río Sauce Chico: Estudio hidrográfico para un desarrollo sustentable. Tesis Doctoral en Geografía. U.N.S. 9 pp.

US Army. (2017). Hydrological Engineers Center-Hydrologic Modeling System HEC HMS (4.2.1) [Programa de computadora]. Estados Unidos. Disponible en: https://www.hec.usace.army.mil/software/hec-hms/downloads.aspx

Van Lynden, G.W.J. \& Oldeman, L. R. (1997). The assessment of the human-induced soil degradation in South and Southeast Asia. International Soil Reference and Information Centre. Wageningen, The Netherlands.

Vázquez, P. \& Zulaica, L. (2011). Cambios en el uso de la tierra del partido de Tandil y principales impactos ambientales. Revista Párrafos Geográficos, Vol 10, №2. 242267.

Vélez Upegui, J. J. \& Botero Gutiérrez, A. (2011). Estimación del tiempo de concentración y tiempo de rezago en la cuenca experimental urbana de la quebrada San Luis, Manizales. Dyna, Medellín. año 78, nro. 164, pp58-77.

Wang, Y., Choi, W. \& Deal, B. M. (2005). Long-term impacts of land-use change on nonpoint source pollutant loads for the St. Louis metropolitan area, USA. Environmental Management, 35(2), 194-205. 